bttp://dx.doi.org/10.21707/gs.v11.n02a03

\title{
Efetividade da gestão do Parque Nacional Serra da Capivara, Piauí, Brasil: uma avaliação temporal
}

\author{
Camila dos Santos Brandão ${ }^{1 *}$, Alexandre Schiavetti ${ }^{2}$
}

\begin{abstract}
${ }^{1}$ Doutoranda pelo Programa de Pós-graduação em Desenvolvimento e Meio Ambiente da Universidade Estadual de Santa Cruz, Rod. Jorge Amado, km 16, 45660-900, Ilhéus, Babia, Brasil.

${ }^{2}$ Docente Pleno do Departamento de Ciências Agrárias e Ambientais da Universidade Estadual de Santa Cruz. Rodovia Jorge Amado, km 16, 45.662900, Ilbéus, Babia, Brasil.

*Autorpara correspondência: milab_biologia@yahoo.com.br
\end{abstract}

Recebido em 08 de dezembro de 2015. Aceito em 23 de novembro de 2016. Publicado em 30 de junho de 2017.

Resumo - Este trabalho teve por objetivo identificar as pressões e ameaças que atingem o Parque Nacional da Serra da Capivara (PNSC) e analisar a efetividade da gestão ao longo de 11 anos, através do método de Avaliação Rápida e Priorização da Gestão das Unidades de Conservação (RAPPAM). Foi observado um aumento na criticidade e no número de pressões e ameaças ao longo dos anos de 2005 até 2016, sendo os incêndios antrópicos, o turismo e recreação e as espécies exóticas invasoras as maiores responsáveis por este aumento. Os temas que apresentaram baixo valor de efetividade foram insumos, em 2005 e processos em 2005 e 2010, e os que apresentaram alto valor foram planejamento, em 2010 e processos e resultados, em 2016. Foi observado um crescimento no valor de efetividade da gestão para o PNSC ao longo do tempo, entretanto o valor sempre esteve dentro da faixa de média efetividade. $\mathrm{O}$ ano de 2016 foi o que apresentou melhor valor de efetividade devido aos módulos planejamento da gestão, processo de decisão e resultados. Por fim destacamos a necessidade de um continuo acompanhamento da efetividade da gestão e das atividades que mais impactam o Parque Nacional Serra da Capivara.

Palavras-chave: Pressões e ameacas; RaPPAM; CaAtinga.

Effectiveness of the management of the Serra da Capivara National Park, Piauí State, Brazil: a temporary EVALUATION

Aвstract - This study aimed to identify the pressures and threats that affect the Serra da Capivara National Park (PNSC) and analyze the effectiveness of management over 11 years by the Rapid Assessment and Prioritization of Protected Areas Management (RAPPAM) method. It was observed an increase in criticality and in the number of pressures and threats over the years 2005 to 2016. The anthropic fires, tourism and recreation, and invasive exotic species were the major factors responsible for this increase. Inputs in 2005 and processes in 2005 and 2010 had a low value of effectiveness, whereas planning in 2010 and processes and results in 2016 had a high value. There was an increase in management effectiveness value for the PNSC over time; however, this value always remained within the range of medium effectiveness. The best effectiveness value was recorded in 2016 due to the management planning modules, decision-making and results. Finally, it is worth noting the need for a continuous monitoring of the management effectiveness and activities that most affect the Serra da Capivara National Park.

Keywords: Pressures AND THREATS; RAPPAM; CAATINGA.

Eficacia de la gestión del Parque Nacional Serra da Capivara, Piauí, Brasil: una evaluación temporal

RESumen - El objetivo de este estudio fue identificar las presiones y amenazas que impactan en el Parque Nacional Serra 
da Capivara (PNSC) y analizar la eficacia de la gestión a lo largo de 11 años a través del método de Evaluación Rápida y Priorización de la Gestión de Áreas Protegidas (RAPPAM). Se observó un aumento de la criticidad y en el número de presiones y amenazas durante los años de 2005 a 2016. Los incendios antrópicos, el turismo y recreación y las especies exóticas invasoras fueron los principales responsables de este aumento. Los insumos en 2005 y los procesos en 2005 y 2010 presentaron baja eficacia, y la planificación en 2010 y los procesos y resultados en 2016 presentaron alta eficacia. Se observó un aumento en el valor de la efectividad de la gestión del PNSC a lo largo del tiempo, pero este valor ha estado siempre dentro del rango de efectividad promedio. El mejor valor de efectividad fue registrado en 2016 debido a los módulos de la planificación de la gestión, la toma de decisiones y los resultados. Por último, destacamos la necesidad de un monitoreo continuo de la efectividad de la gestión y de las actividades que más impactan el Parque Nacional Serra da Capivara.

Palabras Clave: Presiones y amenazas; RAPPAM; CaAtinga.

\section{INTRODUÇÃO}

No ano 2000 foi instituído o Sistema Nacional de Unidades de Conservação da Natureza (SNUC), através da Lei 9.985 (Brasil 2000), representando um fortalecimento na capacidade de planejar e manejar as unidades de conservação (UC) (Silva 2005). As unidades de conservação do Brasil foram divididas em 12 categorias, dentro das classes de Proteção Integral, que tem por função a manutenção dos ecossistemas livres de alterações causadas por interferência humana, e de Uso Sustentável, na qual é permitida a exploração do ambiente de maneira a garantir a perenidade dos recursos ambientais renováveis (Brasil 2000).

Entre as categorias de Proteção Integral tem-se os Parques Nacionais (PARNA), que objetivam preservar os ecossistemas naturais de grande relevância ecológica e beleza cênica, permitindo a realização de pesquisas científicas e atividades de educação ambiental, recreação e turismo ecológico (Brasil 2000). Dos 62 Parques Nacionais brasileiros, o Parque Nacional Serra da Capivara (PNSC), se destaca pela importância cultural e ambiental, devido a registros da presença humana de mais de 50.000 anos atrás, além de apresentar fauna e flora características do bioma caatinga (Oliveira Filho e Monteiro 2009).

Entretanto, as UCs no Brasil apresentam uma série de problemas na gestão, como a deficiência na fiscalização das atividades ilegais (Brockelman e Griffiths 2002), situação fundiária indefinida, com a presença de populações residindo dentro de muitos parques (Rocha et al. 2010), além de recursos humanos e financeiros insuficientes (Schiavetti et al. 2012) e precariedade no processo de implementação (Lemos de Sá e Ferreira 2000), levando ao surgimento do que Terborgh e Schaik (2002) chamam de "unidades de papel", devido ao não cumprimento efetivo dos objetivos para os quais foram criadas.

Dentro desta perspectiva estudos que avaliem a efetividade da gestão das unidades de conservação têm sido essenciais por fornecerem informações uteis para auxiliar os gestores e tomadores de decisões (Day et al. 2012), identificando os pontos fortes e fracos da gestão, e as pressões e ameaças que atingem as UCs (Leverington et al. 2010). Dentre os métodos de avaliação da efetividade, um dos mais aceitos é a Avaliação Rápida e Priorização da Gestão das Unidades de Conservação (RAPPAM), proposto por Ervin (2003), estando de acordo com o referencial proposto pela a Comissão Mundial de Áreas Protegidas (WCPA/IUCN).

No mundo, o questionário RAPPAM já foi aplicado em mais de 53 países e 1.600 unidades de conservação desde o ano de 2003 (Leverington et al. 2010). Já no Brasil este questionário foi aplicado pela primeira vez em 2004, no Estado de São Paulo (Fecuri 2004), sendo que no ano de 2005 iniciou-se o ciclo de aplicação do 
RAPPAM nas UCs Federais (Ogana e Drumond 2007), totalizando 473 unidades de conservação no Brasil analisadas por este método, entre Federais e Estaduais, até o ano de 2011 (Ogana et al. 2012). Deste modo, este trabalho teve por objetivo analisar as pressões e ameaças e a efetividade da gestão doParque Nacional da Serra da Capivara ao longo de 11 anos, avaliados por três aplicações do método RAPPAM (2005, 2010 e 2016).

\title{
Metodologia
}

\author{
Área de estudo
}

O Parque Nacional Serra da Capivara (PNSC) foi criado em 05 de junho de 1979 para proteger os mais de 900 sítios arqueológicos encontrados na região (Pessis e Guidon 2007). Está situado na região sudeste do estado do Piauí, Nordeste do Brasil, ocupando os municípios de São Raimundo Nonato, São João do Piauí, Coronel

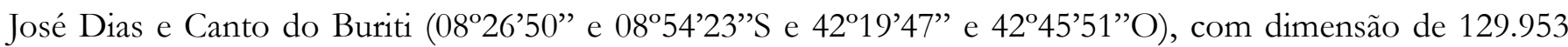
ha (FUMDHAM 1998). O Parque está localizado no bioma caatinga, com vegetação caducifólia e fisionomia arbustiva, com alguns elementos arbóreos e apresenta diversas espécies ameaçadas de extinção.

O PNSC apresenta grande importância para o Brasil e para o mundo devido aos registros de presença humana de mais de 50.000 mil anos (Oliveira Filho e Monteiro 2009; FUMDHAM 2016) e por concentra a maior parte da arte pré-histórica dos primeiros povos que habitaram as Américas, tendo sido reconhecido pela UNESCO, em 1991, como um dos Patrimônios Mundiais da Humanidade (FUMDHAM 2016), e inscrito no livro de Tombo arqueológico, etnográfico e paisagístico, pelo IPHAN, como patrimônio Nacional em 1993.

\section{Material e método}

O questionário RAPPAM é composto por aproximadamente 100 questões de múltipla escolha e por uma lista de 16 pressões e ameaças. A obtenção dos dados do questionário RAPPAM, nos anos de 2005 e 2010, aplicados por meio de oficinas aos gestores e analistas ambientais das UCs Federais, pelo Instituto Chico Mendes de Conservação da Biodiversidade (ICMBio) e WWF-Brasil, foi realizado através do site do WWF-Brasil (2016). Para o ano de 2016, a obtenção dos dados se deu através do envio do mesmo questionário à gestora do Parque, através de e-mail, no mês de abril de 2016, após um contato inicial para explicar sobre o desenvolvimento da pesquisa e após aprovação do Sistema de Autorização e Informação em Biodiversidade (SISBIO no 53397-1).

Definiu-se pressões como as atividades que aconteceram nos últimos 5 anos na unidade de conservação, e como as ameaças as atividades potenciais para acontecerem nos próximos 5 anos (Ervin 2003). O módulo de pressão e ameaça foi analisado com base na tendência, abrangência, impacto e permanência de cada uma das 16 atividades. A tendência indica os aumentos e reduções de cada atividade ao longo do tempo, variando de $-2 \mathrm{a}+2$. A abrangência, o impacto e a permanência possuem pontuação variando de $1 \mathrm{a}$ 4, de acordo com a intensidade, e a multiplicação sucessiva destes elementos indica o grau de cada pressão e ameaça, com pontuação máxima de 64 pontos.

Para verificar a criticidade das pressões e ameaças nos questionários aplicados em 2005, 2010 e 2016, foi feito a soma dos graus de todas as pressões e ameaças em cada ano. Este valor foi dividido pela soma da pontuação máxima possível de 1024 pontos (16 atividades), para se obter em porcentagem a criticidade das 
pressões e ameaças.

A avaliação dos temas Contexto, Planejamento, Insumos, Processos e Resultados, e dos seus respectivos módulos no questionário RAPPAM foi feita através das respostas dadas para as questões, que apresentam 4 opções (sim, predominantemente sim, predominantemente não e não), com a pontuação variando do 0 ao 3 , sendo 0 para não e 3 para sim (Quadro 1).

Quadro 1 - Temas, módulos e número de questões, para os anos pesquisados, no questionário de Avaliação Rápida e Priorização do Manejo de Unidades de Conservação (RAPPAM).

\begin{tabular}{|c|c|c|c|}
\hline \multirow{2}{*}{ Temas } & \multirow{2}{*}{ Módulo } & \multicolumn{2}{|c|}{ Número de Questões } \\
\hline & & 2005 & 2010 e 2016 \\
\hline \multirow{3}{*}{ Contexto } & 1. Importância Biológica & 10 & 9 \\
\hline & 2. Importância Socioeconômica & 10 & 9 \\
\hline & 3. Vulnerabilidade & 9 & 10 \\
\hline \multirow{3}{*}{ Planejamento } & 4. Objetivos & 5 & 6 \\
\hline & 5. Amparo Legal & 5 & 5 \\
\hline & 6. Desenho & 6 & 7 \\
\hline \multirow{4}{*}{ Insumos } & 7. Recursos Humanos & 5 & 5 \\
\hline & 8. Comunicação e Informação & 6 & 6 \\
\hline & 9. Infraestrutura & 5 & 5 \\
\hline & 10. Recursos Financeiros & 6 & 6 \\
\hline \multirow{3}{*}{ Processos } & 11.Planejamento & 5 & 5 \\
\hline & 12.Processos de Tomada de Decisão & 6 & 8 \\
\hline & 13.Pesquisa e Avaliação & 6 & 7 \\
\hline Resultados & 14. Resultados & 12 & 13 \\
\hline TOTAL & & 96 & 101 \\
\hline
\end{tabular}

Para determinar a porcentagem de efetividade de cada módulo do questionário foi feito uma relação entre a pontuação obtida através das respostas informadas e a pontuação máxima de cada módulo, sendo este valor multiplicado por 100. Para obter a efetividade dos temas do questionário, foi feito a média da porcentagem dos módulos inseridos em cada tema. Já para avaliar a efetividade da gestão no Parque foi feito uma média da porcentagem dos módulos de 4 a 14 (totalizando 11 módulos). Esta porcentagem obtida foi comparada aos valores padrões para averiguar a situação de manejo que se encontra o PNSC em cada ano (>40\% baixo; 40-60\% médio; $<60 \%$ alto). Por fim foi empregado Análise de Componente principais (PCA) para explorar as relações entre os módulos dos questionários aplicados e os anos pesquisados.

\section{REsultados}

A análise da criticidade das pressões e ameaças que atingem o PN Serra da Capivara, do ano de 2005 até 2016, mostrou um aumento de valor ao longo dos anos, com a criticidade em 2016 (10,5 e 12,5, para as pressões e ameaças, respectivamente) sendo maior que o triplo do valor de 2005 (3,4 e 3,9, para as pressões e ameaças, respectivamente). Entre as 16 pressões e ameaças possíveis de ocorrer no Parque Nacional Serra da Capivara, 4 não foram identificadas em nenhum ano da pesquisa (pesca, expansão urbana, presença de população humana e coleta de produtos não madeireiros) (Figura 1).

Dentre as pressões, o ano de 2010 foi o que apresentou o maior valor de pontuação (caça); o ano de 2005 foi o que possuiu menor número de pressões (8 pressões); e em 2016 foram observados 2 pressões a mais que nos outros anos (disposição de resíduos e processos seminaturais) (Figuras 1a). Já para as ameaças, o ano de 2010 também foi o que apresentou maior valor de pontuação (caça), e o ano de 2005 o que apresentou o menor número de ameaças (7 ameaças), entretanto este ano também apresentou alto valor de pontuação (construção de 
infraestrutura); e em 2016 foram observadas 3 ameaças a mais (disposição de resíduos, processos seminaturais e extração de madeira) que nos outros anos (Figuras 1b).

Figura 1 - Número de pressões (a) e ameaças (b) e o valor de pontuação nos anos de 2005, 2010 e 2016 para o Parque Nacional Serra da Capivara (Piauí, Brasil).
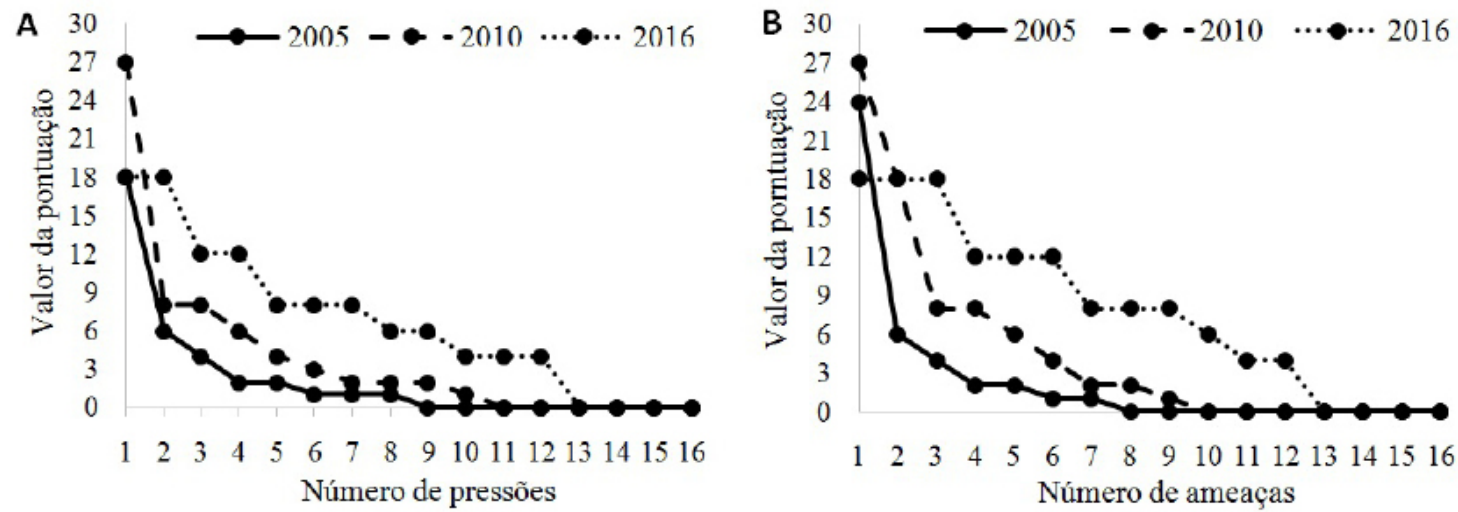

Quanto a tendência das pressões e ameaças, foi observado uma diminuição para as pressões, com o somatório das pontuações atingindo -1 em 2005, -4 em 2010, -3 pontos em 2016. Já para as ameaças foi observada uma tendência de aumento para o ano de 2005 e 2010 (4 e 6 pontos, respectivamente) e de redução para 2016 (-4 pontos).

A Tabela 1 evidencia os valores de efetividade dos módulos do questionário RAPPAM durante a pesquisa. Os módulos de importância biológica, importância socioeconômica e infraestrutura apresentaram altos valores de efetividade nos 3 anos analisados (>60\%). O módulo objetivo apresentou alto valor para os anos de 2005 (66,67\%) e 2016 (77,78\%); os módulos amparo legal e desenho demonstraram alto valor no ano de 2010 (73,33\%, e 66,67\%, respectivamente) e os módulos vulnerabilidade, processo de gestão e resultado apresentaram alto valor de efetividade para 2016 (66,67\%, 70,83\% e 79,49\%, respectivamente).

A Tabela 1 também apresenta a diferença entre os valores dos módulos de 2005 e 2016. Apenas os módulos de importância biológica $(-19,63)$ e amparo legal $(-20,00)$ apresentaram redução entre os anos. O módulo resultado foi o que exibiu maior aumento entre os anos $(35,04)$, seguido do planejamento da gestão (33,33), vulnerabilidade $(29,63)$ e processo de decisão $(26,39)$.

Tabela 1 - Efetividade dos módulos do questionário RAPPAM nos anos da pesquisa, e diferença percentual entre os anos de 2005 e 2016, no Parque Nacional Serra da Capivara, Piauí, Brasil.

\begin{tabular}{ccccc}
\hline MÓDULOS & $\mathbf{2 0 0 5}$ & $\mathbf{2 0 1 0}$ & $\mathbf{2 0 1 6}$ & Diferença Percentual \\
\hline Importância Biológica & 90,00 & 85,19 & 70,37 & $-19,63$ \\
Importância Socioeconômica & 63,33 & 70,37 & 77,78 & 14,44 \\
Vulnerabilidade & 37,04 & 36,67 & 66,67 & 29,63 \\
Objetivo & 66,67 & 50,00 & 77,78 & 11,11 \\
Amparo Legal & 46,67 & 73,33 & 26,67 & $-20,00$ \\
Desenho & 44,44 & 66,67 & 66,67 & 22,22 \\
Recursos Humanos & 13,33 & 46,67 & 40,00 & 26,67 \\
Comunicação e Informação & 33,33 & 44,44 & 55,56 & 22,22 \\
Infraestrutura & 60,00 & 73,33 & 66,67 & 6,67 \\
Recursos Financeiros & 27,78 & 27,78 & 33,33 & 5,56 \\
Planejamento da Gestão & 26,67 & 20,00 & 60,00 & 33,33 \\
Processo de Decisão & 44,44 & 37,50 & 70,83 & 26,39 \\
Pesquisa e Avaliação & 38,89 & 47,62 & 52,38 & 13,49 \\
Resultados & 44,44 & 43,59 & 79,49 & 35,04 \\
\hline
\end{tabular}


Dentre os temas do questionário RAPPAM, o planejamento, no ano de 2010, e os processos e resultados no ano de 2016, apresentaram alto valor de efetividade (>60\%) (Figura 2). Além disto, o tema insumos, em 2005 e o tema processos em 2005 e 2010 apresentaram baixo valor de efetividade ( $<40 \%)$. Quanto a efetividade da gestão do Parque Nacional Serra da Capivara, é observado um aumento continuo do valor do ano de 2005 até o ano de 2016, porém, ficando sempre dentro da categoria de média efetividade (Figura 2).

Figura 2 - Efetividade dos temas e da gestão para o Parque Nacional Serra da Capivara (Piauí, Brasil), nos anos pesquisados (acima da linha tracejada - alta efetividade; entre a linha tracejada e continua - média efetividade; abaixo da linha continua - baixa efetividade).

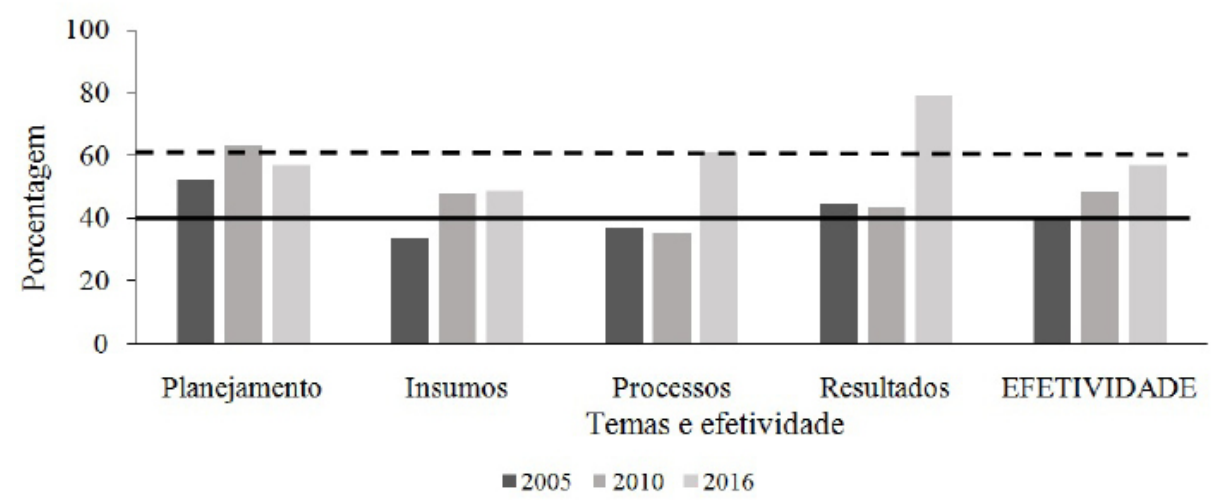

Através da PCA foi possível observar que os 2 primeiro eixos juntos explicam 93,54\% dos dados (Eixo $1=72,87 \%$; Eixo 2 = 21,13\%) (Figura 3). O eixo 1 é justificado pelos módulos recursos humanos e desenho, tendo maior ação sobre o valor de efetivadade da gestão do ano de 2010. Já os módulos planejamento da gestão, processo de decisão e resultados estão mais relacionados ao eixo 2, agindo sobre o valor de efetividade da gestão do ano de 2016. Além disto, também é possível observar, pela analise da Figura 3, que o ano de 2005 tem sua efetividade reduzida pela falta de recursos humanos, enquanto a efetividade para o ano de 2016 está relacionada ao planejamento e aos processos de decisão.

Figura 3 - Análise de componentes principais dos módulos de efetividade de manejo do Parque Nacional Serra da Capivara (Piauí, Brasil) ao longo dos anos 2005, 2010 e 2016.

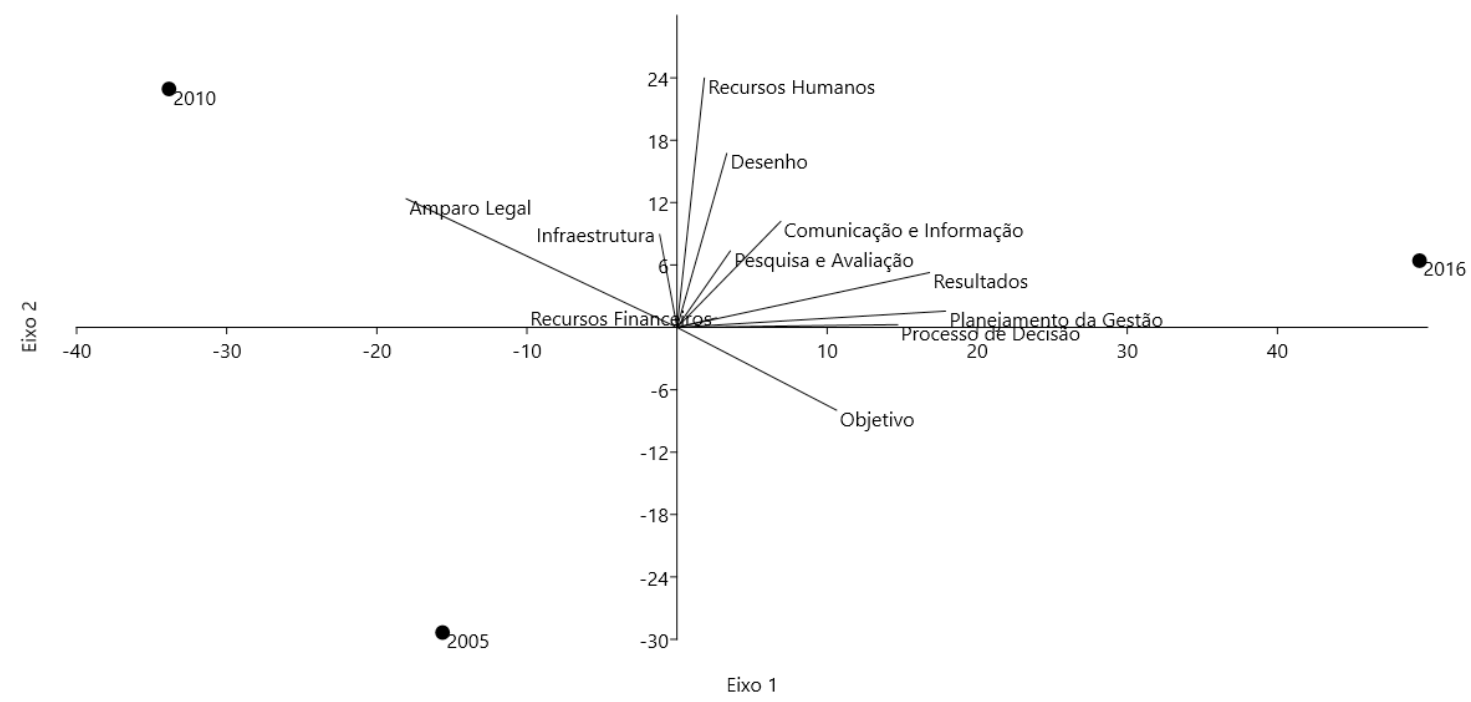




\section{Discussão}

As pressões e ameaças que atingem o Parque Nacional Serra da Capivara apresentaram um aumento do ano de 2005 para 2016, tanto na pontuação quanto na quantidade de atividades (Figura 1). As atividades que mais contribuíram para este aumento e que necessitam de uma maior atenção por parte do gestor e do governo quanto ao estabelecimento de medidas prioritárias para a conservação são turismo e recreação, espécies exóticas invasoras, processos seminaturais (processos naturais que foram intensificados pela intervenção antrópica), influências externas e incêndios antrópicos. A atividade de turismo e recreação merece maior atenção devido a inauguração do aeroporto internacional de São Raimundo Nonato, em novembro de 2015, que teve por intenção facilitar o acesso e aumentar o fluxo de turistas no Parque, que é de aproximadamente 18 mil visitantes por ano, gerando mais emprego e renda para a população do entorno, mas não para o parque, visto que todo o dinheiro arrecado com a venda de ingressos é enviada para o fundo federal (Melina Rangel, Gestora PNSC, Comunicação Pessoal). Por sua vez, a alta atividade de caça que ocorreu no ano de 2010 pode estar relacionada à atuação do gestor responsável à época do levantamento das informações, já que o mesmo não atuava na fiscalização ambiental, e portanto na atividade de caça.

Este mesmo padrão de aumento das atividades têm sido observado para os 64 Parques Nacionais Federais do Brasil, entre os anos de 2005 e 2010, com as pressões saindo de 7,0\% para 12,7\%, e as ameaças saindo de 7,0\% para 13,4\% (ICMBio e WWF-Brasil 2011), sendo pastagem, caça, espécies exóticas invasoras, uso dos recursos pela população, influencias externas e incêndios de origem antrópica as atividades que mais aumentaram. Já no mundo, os principais problemas que afetam os Parques Nacionais, categoria II da Internacional Union for the Conservation of Nature (IUCN), são caça ilegal, extração de madeira e mudança no uso da terra, em 13 Parques na Romênia (Steindlegger e Stanciu 2006) e pastoreio, mudança do uso da terra, processos seminaturais e caça, para 19 Parques na Mongólia (Batsukh e Belokurov 2005).

A análise da tendência das pressões e ameaças ao longo tempo mostrou um panorama controverso, uma vez que foi previsto uma tendência de aumento para as ameaças nos próximos 5 anos após a aplicação do questionário, e foi observado uma tendência de diminuição nas pressões que afetam o PNSC. Esta tendência de maior previsão das ameaças em relação ao observado nas pressões também foi encontrado em todas as categorias de unidades de conservação Federais do Brasil (Ogana e Drumond 2007; Ogana et al. 2012). Isto demonstra que a expectativa do órgão gestor, quanto às ameaças que afetam as UCs no Brasil, está sendo pior do que a realidade, provavelmente devido a uma perspectiva de aumento das atividades proibidas nas unidades em contraste com a redução de recursos financeiros e humanos para auxiliar na fiscalização e prevenção destas ameaças.

Foi observada uma redução da importância biológica do Parque ao longo dos anos, mesmo com a presença de espécies ameaçadas de extinção como urubu-rei (Sarcoramphus papa) e arara-vermelha (Ara chlorptera), provavelmente devido à alta valorização do Parque apenas como local histórico, com a presença dos sítios arqueológicos e das pinturas rupestres, tanto pela mídia, quanto pelos moradores e guias do Parque, além da pouca importância dedicada à caatinga, frente aos outros biomas brasileiros, o que poderia influenciar na visão do gestor. Já a importância socioeconômica do PNSC apresentou crescimento ao longo dos anos, devido ao desenvolvimento da cidade, através do turismo e ao aumento do número de atividades realizadas pela população do entorno, gerando renda para os moradores, através da venda de produtos artesanais, e para os guias do Parque 
(Aguiar e Carvalho 2012) (Tabela 1). Contrariamente ao observado para o PNSC, o módulo de importância socioeconômica apresentou uma redução de 2,0\% do ano de 2005 para 2010, para todas as categorias de UCs investigada no questionário RAPPAM, e uma redução de 1,8\% para os Parques Federais analisados (Ogana e Drumond 2007; Ogana et al. 2012).

Um módulo que merece atenção especial é a vulnerabilidade, uma vez que o aumento do valor de efetividade deste módulo ao longo do tempo apresenta relação inversa com o estado de conservação da unidade. Para o PNSC este módulo saiu de 37,04\% em 2005 para 66,67\% em 2016, totalizando um aumento de 29,63 pontos (Tabela 1). Este aumento foi devido, principalmente, a problemas relacionados às práticas culturais, crenças e usos tradicionais que estão em conflito com os objetivos do Parque e devido a existência de uma grande demanda, por parte da população do entorno, por recursos naturais encontrados na UC. Resultado inverso foi obtido para os Parques federais do Brasil, que apresentaram uma redução deste módulo de 7,2 pontos entre os anos de 2005 e 2010, e para as 292 UCs Federais, de diversas categorias, que apresentaram uma redução de 5,5 pontos (ICMBio e WWF-Brasil 2011).

Dentre os temas de efetividade da gestão o planejamento foi o que apresentou maior valor médio entre os anos, devido a melhora no módulo objetivo, e o tema insumos o que apresentou o menor valor médio, com maior influência do módulo recursos financeiros, afetando o valor de efetividade da gestão do PNSC. Nos Parques Federais do Brasil o tema que apresentou maior valor de efetividade também foi planejamento $(60,8 \%$, em 2010), com o módulo objetivo apresentando maior valor; já o tema com menor valor foi os insumos (40,6\%, em 2010), com os módulos recursos financeiros e humanos possuindo menor valor de efetividade (Ogana e Drumond 2007; ICMBio e WWF-Brasil 2011; Ogana et al. 2012). Também foi obtido o tema planejamento como maior, e os temas resultados e insumos como os menores para os Parques da Mongólia e da Romênia, respectivamente (Batsukh e Belokurov 2005; Steindlegger e Stanciu 2006).

O módulo objetivo quase sempre apresentou altos valores de gestão, devido aos objetivos de criação do PNSC terem sido bem estabelecidos e serem de conhecimento do órgão gestor e das comunidades locais, o que afetou positivamente este módulo. Por outro lado, Leverington et al. (2010) destacam as baixas pontuações nas perguntas sobre recursos humanos e financeiros, do tema insumos, como um padrão global, sendo isto observado em vários estudos pelo mundo (Gerhardinger et al. 2011; Lu et al. 2012; Magris et al. 2013). O PNSC também segue este padrão, possuindo um quadro de funcionários insuficientes para a realização das atividades de conservação do Parque, sendo observada uma melhora na capacitação dos funcionários, mas não na quantidade ao longo do tempo da pesquisa, o que afetou negativamente este módulo. Vale destacar que a Fundação Museu do Homem Americano (FUMDHAM) também conta com um quadro de funcionários que auxiliam nas atividades do Parque, e que não foram contabilizados na pesquisa, mas que segundo informações prestadas, também se encontra em número insuficiente para a realização das atividades conservação, como a limpeza e manutenção das pinturas do Parque.

O PARNA Serra da Capivara, no ano de 2015, possuiu um orçamento anual de $\mathrm{R} \$ 2.694 .395,44$, para uma área de aproximadamente 100.000 há, não sendo suficiente para a realização de todas as suas atividades, como a manutenção das estradas, a compra de água para as instalações do Parque e para os bebedouros dos animais, o pagamento dos funcionários da guarita, fiscais e de limpeza e para a manutenção das estruturas físicas e das pinturas e sítios arqueológicos no Parque. Entretanto, esta situação apresenta uma perspectiva de melhora no futuro, devido a Justiça Federal ter obrigado a União, o Ibama e o Instituto do Patrimônio Histórico e Artístico 
Nacional (Iphan) o repasse de R \$4.493.145,00, no início do ano de 2016, destinados a manutenção, conservação e preservação do Parque (G1 2016). A mesma decisão judicial também obrigou o ICMBio a elaborar o plano de planejo, inexistente no Parque, mesmo depois de 37 anos de criação, no período máximo de um ano. Deste valor, apenas $\mathrm{R} \$$ 3.8000.000,00 foram encontrados e retidos das contas da união, e até o mês de julho de 2016 ainda não havia sido feito o repasse para a gestão do Parque (Melina Rangel, Gestora PNSC, Comunicação Pessoal). Esta verba será gerida em parceria com a FUMDHAN, que possui um sistema de cooperação com o Parque, auxiliando nas diversas atividades de monitoramento e preservação dos sítios arqueológicos e das pinturas rupestres.

O Brasil foi um dos países a assinar a meta da Convenção da Diversidade Biológica (CBD), que determina até o ano de 2020, o prazo para criação de mais unidades de conservação e a que as mesmas sejam efetivas na proteção dos seus ambientes (IUCN et al. 2011). Esta pesquisa encontrou uma melhora no valor de efetividade da gestão do PNSC ao longo dos anos, estando este valor sempre dentro da faixa de média efetividade (Figura 2). Nas UCs Federais brasileiras avaliadas pelo ICMBio também foi observado esta melhora no valor de efetividade, tanto para os Parques como para as demais categorias de unidade de conservação (Ogana e Drumond 2007; ICMBio e WWF-Brasil 2011; Ogana et al. 2012), com esta melhora estando, provavelmente, associada à criação de um órgão responsável apenas pela proteção das espécies da fauna e da flora e do estabelecimento do Sistema Nacional de Unidades de Conservação, o ICMBio, criado no ano de 2007 (Brasil 2007).

A PCA possibilitou a observação de que o ano de 2016, que apresentou maior valor de efetividade entre os anos pesquisados, foi influenciado mais fortemente pelos módulos planejamento da gestão, processo de decisão e resultados (Figura 3). Isto fica evidente uma vez que ocorreu uma grande melhora dos temas processos e resultados dos anos de 2005 e 2010, para o ano de 2016 (Figura 2). O módulo resultados aborda os produtos e serviços realizados nos últimos 2 anos pelos funcionários, gestores, voluntários e membros da comunidade da UC, tendo sido observado uma melhora principalmente quanto a recuperação de áreas e ações mitigatórias realizadas. Quanto ao módulo planejamento da gestão, parte da melhora para o ano de 2016 se deu devido a ação do gestor, o sr. Fernando Tizianel, que atuou entre o período de 2011 e 2014, e teve uma forte ação nas questões do planejamento da unidade, e a outra parte foi devido à mudança do gestor, como observado para a pergunta sobre a existência de plano de manejo, inexistente no Parque, mas pontuado de forma alta no ano de 2016. Esta grande variação no valor do ano de 2016, provavelmente devido a mudança de gestor, também foi observada para o módulo amparo legal, que apresentou redução neste ano.

Esta mudança frequente de gestor é observada não apenas para o PARNA Serra da Capivara, que durante o período da pesquisa possuiu pelo menos 4 gestores diferentes, mas também para a maioria das UCs brasileiras, afetando o conhecimento acerca da unidade e, por conseguinte, as informações prestadas necessárias para executar a metodologia RAPPAM. Dentro desta perspectiva, Ervin (2003) argumenta sobre a qualidade das informações prestadas pelos gestores e administradores, que precisam ser adequadas para prover dados suficientes para a análise, implicando no valor de efetividade da gestão. Além disto, outras limitações desta metodologia são apresentadas por Veenvliet e Sovinc (2009) como o questionário não permitir uma análise aprofundada das respostas, obtendo-se apenas o valor final de efetividade, e ignorando as perguntas com respostas negativas, que merecem mais atenção por parte da gestão; não existir a opção de resposta “não conheço/sei” no questionário; e existir questões duplamente negativas, que podem induzir a um mal entendimento do sentido da pergunta por parte do respondente.

Acompanhar a evolução das pressões e ameaças e da efetividade do Parque Nacional Serra da Capivara tem 
uma importância ainda maior, devido ao bioma onde esta unidade se encontra, a caatinga. Este bioma representa $11,0 \%$ do território nacional, sendo que destes aproximadamente $46 \%$ sofrem devido ao desmatamento, e apenas cerca de 7,5\% está dentro de algum tipo de unidade de conservação (MMA 2016). Já no Estado do Piauí, onde se encontra o PNSC, a caatinga representa 37\% do território, com o Parque possuindo 75\% de cobertura vegetal com este bioma (PLANAP, 2006). As UCs da caatinga, assim como o PNSC, vêm apresentando aumento no valor de efetividade ao longo do tempo, com melhora de 3,2\% entre os anos de 2005 e 2010, mantendose sempre dentro da categoria de média efetividade, devido a uma melhora nos módulos recursos humanos, pesquisa e avaliação e desenho e no tema resultados (Ogana e Drumond 2007; ICMBio e WWF-Brasil 2011; Ogana et al. 2012). Além disto, estes autores encontraram um aumento do nível de criticidade, para o bioma caatinga, entre os anos de 2005 e 2010 de 8,3\%, assim como nesta pesquisa.

\section{Agradecimentos}

Os autores gostariam de agradecer a antiga Gestora do PNSC, Sra. Maria Lucia Carvalho, por se disponibilizar a responder o questionário, a atual gestora Melina Rangel, pelas informações prestadas a posteriori e ao ICMBio pela autorização à pesquisa. Também gostaríamos de agradecer à bolsa de doutorado fornecido pela Coordenação de Aperfeiçoamento de Pessoal de Nível Superior (CAPES) à primeira autora e à bolsa de produtividade CNPq fornecida ao último autor.

\section{CONCLUSÃo}

Com este trabalho foi possível conhecer a situação de efetividade do Parque Nacional Serra da Capivara, sendo observada uma pequena melhora ao longo do tempo. Os módulos que merecem uma maior atenção por parte da gerência do Parque, por serem mais críticos e afetarem negativamente o valor de efetividade, para o ano de 2016, foram recursos humanos, pontuado como insuficientes pelos gestores em todos os anos da pesquisa e recursos financeiros. Além disto, o módulo vulnerabilidade também merece atenção, devido ao aumento da demanda, pela população do entorno, dos recursos naturais encontrados no PNSC. Por sua vez as principais atividades que podem atingir esta unidade nos próximos anos e que por isto merecem maior atenção quanto as ações de monitoramento e fiscalização são turismo e recreação e incêndios antrópicos.

Por fim, os autores desta pesquisa destacam a importância da continuação de pesquisas que visem avaliar a efetividade da gestão, tanto para o PNSC, como para as demais unidades do bioma caatinga, em um período de 5 em 5 anos, através do método de Avaliação Rápida e Priorização da Gestão das Unidades de Conservação (RAPPAM), devido a estruturação do método que permite a obtenção dos dados de forma rápida e prática, para, assim, poder ser feito um acompanhamento das áreas da gestão que precisam de maior atenção, com o intuito de tornar a unidade efetiva em cumprir os objetivos para a qual foi criada. 


\section{REFERÊNCIAS}

Aguiar EPS, Carvalho SMS. 2012. Turistas de São Raimundo Nonato - PI: perfil e grau de satisfação quanto aos serviços de alimentação. TurismoemAnálise, 23(3):600-622.

Batsukh N, Belokurov A. 2005. Mongolia: Management Effectiveness Assessment of the Mongolian Protected Areas System using WWF's RAPPAM Methodology. Gland, Switzerland: WWF, 46 p.

Brasil. Lei 11.516 de 2007. Cria o Instituto Chico Mendes de Conservação da Biodiversidade. 2007. http://www.planalto.gov.br/ccivil 03/ ato20072010/2007/lei/111516.htm. (Acesso em: mai. 2016).

Brasil. Sistema Nacional de Unidades de Conservação. Lei no 9.985, de 18 de julho de 2000. 2000. http:// www.planalto.gov.br/ccivil/leis/L9985.htm. (Acesso em: mai. 2016).

Brockelman WY, Griffiths M. 2002. Mecanismos de fortalecimento das áreas protegidas. In: Terborgh J, Schaik CV, Davenport L, Rao M. (Org.). Tornando os parques eficientes: estratégias para conservação da natureza nos trópicos. Curitiba: Universidade Federal do Paraná, p. 290-304.

Day J, Dudley N, Hockings M, Holmes G, Laffoley D, Stolton S, WellsS.2012.Guidelines for applying the IUCN Protected Area Management Categories to Marine Protected Areas. Gland, Switzerland: IUCN, $36 \mathrm{p}$.

Erol SY, Kuvan Y, Yildirim HT. 2011.The general characteristics and main problems of national parks in Turkey. African Journal of Agricultural Research,6(23):5377-5385.

Ervin J. 2003. WWF rapid assessment and prioritization of protected area management (Rappam) methodology. Gland, Swizertland: WWF, 52 p.

Fecuri J. (Org.) 2004. Implementação do RAPPAM em Unidades de Conservação do Instituto Florestal e da Fundação Florestal de São Paulo. São Paulo: WWF-Brasil, Instituto Florestal, 44 p.

FUMDHaM - Fundação Museu do Homem Americano. 1998. Parque Nacional Serra da Capivara. São Raimundo Nonato/Piauí: FUMDHAM/ FNMA/ASMOCO. Alínea Publicações Editora,94 p.

FUMDHaM - Fundação Museu do Homem Americano. Parque Nacional Serra da Capivara. 2016.http:// www.fumdham.org.br. (Acesso em: abr. 2016).

G1. Decisão judicial. 2016.http://g1.globo.com/pi/piaui/noticia/2016/02/juiz-determina-repasse-de-r-44milhoes-para-serra-da-capivara.html.(Acesso em: abr. 2016).

Gerhardinger LC, Godoy EA, Jones PJ, Sales G, Ferreira BP. 2011.Marine Protected Dramas: The Flaws of the Brazilian National System of Marine Protected Areas. Environmental Management, 47(4):630-643.

ICMBio, WWF-Brasil. 2011.Avaliação comparada das aplicações do método RAPPAM nas unidades de conservação federais, nos ciclos 2005-06 e 2010. Brasília: ICMBio, 137 p. 
Lemos de Sá RM, Ferreira LM. 2000.Áreas protegidas ou espaços ameaçados: o grau de implementação e a vulnerabilidade das Unidades de Conservação federais brasileiras de uso indireto. Brasília, DF: WWF-Brasil, 32 p.

Leverington F, Costa KL, Courrau J, Pavese H, Nolte C, Marr M, Coad L, Burgess N, Bomhard B, Hockings M. 2010. Management Effectiveness Evaluation in Protected Areas-A Global Study. 2 ed.Brisbane: University of Queensland, 101 p.

Lu DJ, Kao CW, Chao CL. 2012. Evaluating the Management Effectiveness of Five Protected Areas in Taiwan Using WWF's RAPPAM. Environmental Management, 50(2):272-282.

Magris RA, Mills M, Fuentes MMPB, Pressey RL. 2013. Analysis of progress towards a comprehensive system of Marine Protected Areas in Brazil. Natureza e Conservação, 11(1):81-87.

MMA - Ministério do Meio Ambiente. Bioma Caatinga. 2016.http://www.mma.gov.br/biomas/ caatinga. (Acesso em: mai. 2016).

Ogana CA, Drumond MA, Ferreira MN. 2012. Efetividade da gestão das unidades de conservação federais do Brasil: resultados de 2010. Brasília: WWF-BRASIL; ICMBio, 84 p.

Ogana CA, Drumond MA. (Org.) 2007. Efetividade de gestão das unidades de conservação federais do Brasil. Brasília: Ibama, 96 p.

Ogana CA. (Rev.) 2014. Implementação da avaliação rápida e priorização da gestão de unidades de conservação (RAPPAM) em unidades de conservação estaduais em Goiás. Goias: WWF, 109 p.

Oliveira Filho RC, Monteiro MSL. 2009. Ecoturismo no Parque Nacional Serra da Capivara: trata-se de uma prática sustentável?. Turismo em Análise, 20(2):230-250.

Pessis A, Guidon N. 2007. Serra da Capivara National Park, Brazil: cultural heritage and society. World Archaeology, 39(3):406-416.

PLANAP - Plano de Desenvolvimento do Parnaiba. 2006. Plano de Ação para o Desenvolvimento Integrado da Bacia do Parnaíba. Brasília, DF: CODEVASF: TODA Desenhos \& Arte Ltda. 76 p.

Rocha LGM, Drummond JÁ, Ganem RS. 2010. Parques nacionais brasileiros: problemas fundiários e alternativas para a sua resolução. Revista de Sociologia e Política, 18(36):205-226.

Schiavetti A, Magro TC, Santos MS. 2012. Implementação das unidades de conservação do corredor central da mata atlântica no estado da Bahia: desafios e limites. Revista Árvore, 36(4):611-623.

Silva M. 2005. O programa brasileiro de unidades de conservação. Megadiversidade, 1(1):22-26.

Steindlegger E, Stanciu G. 2006.RAPPAM (Rapid Assessment and Prioritization of Protected Area Management): methodology implementation in Romania. Romania: WWF, 50 p.

Terborgh J, Schaik CV. 2002. Por que o mundo necessita de parques? In: Terborgh J, Schaik CV, Davenport L, 
Rao M (Org.)Tornando os Parques eficientes: estratégias para a conservação da natureza nos trópicos. Curitiba: Universidade Federal do Paraná, p. 25-36.

Veenvliet JK, Sovinc A. 2009. Protected area management effectiveness in Slovenia, Final report of the RAPPAM analysis. Slovenia: WWF, 56 p.

WWF-Brasil, Observatório de UCs. 2016. http://observatorio.wwf.org.br/unidades. (Acesso em: fev. 2016). 